\title{
PREDICTION OF BLENDED YARN EVENNESS AND TENSILE PROPERTIES BY USING ARTIFICIAL NEURAL NETWORK AND MULTIPLE LINEAR REGRESSION
}

\author{
Samander Ali Malik ${ }^{*}$, b, Assad Farooq ${ }^{c}$, Thomas Gereke ${ }^{b} \&$ Chokri Cherif ${ }^{b}$ \\ a Department of Textile Engineering, Mehran University of Engineering \& Technology, 76062 Jamshoro, Sindh, Pakistan \\ ${ }^{\mathrm{b}}$ Institute of Textile Machinery and High Performance Material Technology, Technische Universität Dresden, 01062 Dresden, Germany \\ 'Department of Fiber and Textile Technology, University of Agriculture Faisalabad, 38000 Faisalabad, Pakistan \\ * Corresponding author: e-mail samander_ali.malik@tu-dresden.de, phone +49 351 46342244, fax +49 35146334026
}

\begin{abstract}
:
The present research work was carried out to develop the prediction models for blended ring spun yarn evenness and tensile parameters using artificial neural networks (ANNs) and multiple linear regression (MLR). Polyester/ cotton blend ratio, twist multiplier, back roller hardness and break draft ratio were used as input parameters to predict yarn evenness in terms of $\mathrm{CVm} \%$ and yarn tensile properties in terms of tenacity and elongation. Feed forward neural networks with Bayesian regularisation support were successfully trained and tested using the available experimental data. The coefficients of determination of ANN and regression models indicate that there is a strong correlation between the measured and predicted yarn characteristics with an acceptable mean absolute error values. The comparative analysis of two modelling techniques shows that the ANNs perform better than the $M L R$ models. The relative importance of input variables was determined using rank analysis through input saliency test on optimised ANN models and standardised coefficients of regression models. These models are suitable for yarn manufacturers and can be used within the investigated knowledge domain.
\end{abstract}

\section{Keywords:}

Blended yarn evenness; tenacity; model; artificial neural network; multiple linear regression; rank analysis

\section{Introduction}

Blended yarns, especially polyester/cotton blends - hereafter called PES/CO - are most widely used in apparel, home furnishing and other applications due to their characteristic properties. It is well known that cotton fibres have a pleasant and comfortable skin touch, while polyester enhances the fabric's useful life because of its strength. The quality of yarn is attributed to its evenness and its tensile properties.

Yarn quality is a complex characteristic and it is influenced by various material and process parameters. Several researchers have investigated blended yarn characteristic properties using different influencing variables. Research reveals that blended yarn quality depends on machine type and machine parameters, material type and its proportion [1-3].

This study was conducted to predict PES/CO blended yarn evenness in terms of yarn irregularity $(\mathrm{CVm} \%)$ and tensile properties using blend ratio, twist multiplier, back roller cot hardness and break draft ratio as influencing variables. In previous research $[4,5]$ the significance of aforementioned parameters on $\mathrm{PES} / \mathrm{CO}$ blended yarn quality and tensile properties was explored experimentally but it is challenging to develop relationship between yarn properties and collective machine and material parameters analytically. As discussed earlier, there exist complex interactions among yarn quality characteristics and machine and material variables, and in such conditions, conventional mathematical techniques which require assumptions and constants are not much suitable to predict yarn properties with high precision.
The motivation behind the use of artificial neural networks (ANNs) lies in their flexibility and power of information processing, because they can solve complex problems by learning the inputoutput patterns provided by the user. ANNs have been used successfully as a predicting tool in all areas of textiles from fibre to complex composites. In textile spinning domain ANNs have been used to detect and classify trash particles in cotton web [6], control of draw frame sliver evenness and levelling action point from machine and material parameters [7], optimisation of spinning process at ring frame using draw frame parameters [8], the prediction of ring spun cotton yarn properties from HVI (high volume instrument) characteristics of fibres [9], comparison of ANN and regression models for yarn hairiness, evenness and tensile properties using fibre HVI properties, roving properties, yarn count and twist multiplier [10, 11]. Apart from ring spinning ANN has also served as a prediction tool for other processes as well. Polyester-viscose rotor spun breaking elongation and evenness was predicted from blend ratio and process parameters $[12,13]$, the prediction of breaking load and breaking elongation of air jet spun yarn from material and process parameters [14], prediction of worsted spinning performance from fibre and machine parameters [15] and determining the spin ability of cotton fibres at rotor spinning using different machine and material parameters as input [16].

In conjunction to cited literature, there is a lack of published literature on the proposed theme of research; most of the developed models are related to cotton fibre spinning especially using fibre HVI properties. Break draft ratio and back roller cot hardness have never been used as predictors though they 
have a significant influence on yarn properties and machine performance. By considering the above note, research theme of prediction of PES/CO blended yarn evenness and tensile properties from fibre blend ratio, twist multiplier, back roller cot hardness and preparatory (back/break) draft is presented. The blend ratio is selected carefully to cover a wide knowledge domain of both fibres, whereas twist multiplier, break draft ratio and back roller cot hardness are also selected according to practical yarn spinning requirements, which is vital for modelling.

\section{Experimental}

\section{Materials}

Polyester and cotton fibres have been selected as input material in the present work due to their dominant share in the apparel and home furnishing markets. In this proposed research work polyester fibres of 1.2 denier (1.33 dtex) fineness and 1.5" (38 mm) staple length and cotton fibres of fineness (mic value) $4.3 \mu \mathrm{g} /$ in (1.69 dtex) and $2.5 \%$ span length (SL) 1.1 " $(28 \mathrm{~mm})$ were used. Before processing into spinning sequence of machines, standard material conditioning procedure was followed. Both fibres were processed separately in blow room and Rieter high performance card C-51, whereas the blending was done at auto levelling draw frame RSB D35. The finisher sliver of 59.5 grains/yard (4.2 ktex) was drafted at simplex FA $415 \mathrm{~A}$ into roving of one hank with twist factor $\left(\alpha_{\mathrm{e}}\right)$ of 35.8. Three blend ratios (PES/CO: 70/30, 50/50 and 30/70) were selected and roving strand was drafted into $\mathrm{Ne} 39.4$ single yarn at ring frame EJM 168. First the machine was optimised for each input parameter for each blend and then keeping optimised parameters as constant other input parameters were altered one by one, as presented in Table 1 (blend ratios have been assigned the coded notations 1-3 for network training). In the present work 48 yarn samples were produced at ring spinning frame with four different input parameters, that is, blend ratio, twist multiplier, back roller cot hardness and break draft.

Table 1. Selected parameters for prediction

\begin{tabular}{|c|c|}
\hline Influencing Variables & Values \\
\hline PES/CO blend ratio $[\%]$ & $70 / 30(1), 50 / 50(2), 30 / 70(3)$ \\
\hline Twist multiplier $\left(\alpha_{\mathrm{e}}\right)[-]$ & $3.3,3.5,3.7,3.9,4.0,4.2,4.5$ \\
\hline Cot hardness $\left[{ }^{\circ}\right.$ Shore] & $70,75,80$ \\
\hline Break draft ratio $[-]$ & $1.35,1.38,1.42,1.45,1.49$, \\
& 1.53 \\
\hline
\end{tabular}

\section{Methods}

The yarn evenness was assessed in a standard testing atmosphere, that is, $65 \pm 2 \%$ relative humidity and $20 \pm 2{ }^{\circ} \mathrm{C}$ temperature at Uster Tester 4 according to ASTM Standard D 1425-96 and tensile testing was done at Uster Tensorapid 4 as suggested in ASTM standard D 2256. Before testing, the yarns were conditioned for 24 hours in standard atmospheric conditions. The acquired results were used for the development of ANN and regression models.

\section{Modelling}

\section{Converting PES/CO blend ratio to numerical variable}

$\mathrm{PES} / \mathrm{CO}$ blend ratio, which is the main predictor variable, comprises the weight percentage of constituent fibres in ratios. For ANN and multiple linear regression (MLR) modelling this type of data is not suitable to use directly for model development and analysis. There are different methods available to convert such data into a suitable form. One direct way is to use dummy variables with required factor levels. Here only one variable occurs in three different ratios: PES/CO blend ratio. The advantage of these levels or factors is that their difference is equal. Therefore, coding these factors to dummy numerical values is the easiest and suitable method for this particular data type. Hence, variable PES/CO blend ratio is coded into numerical values as PES/CO 70/30 $=1, \mathrm{PES} / \mathrm{CO} 50 / 50=2$ and $\mathrm{PES} / \mathrm{CO} 30 / 70=3$. This means the higher the proportion of cotton fibre, the greater the coding number and vice versa.

\section{Artificial Neural Network Training}

Because of their adaptive nature, ANNs are commonly used to solve any complex nonlinear problem otherwise not easy to describe with any analytical functions. ANN structure comprises three parts, input neurons which take inputs for network, hidden neurons which perform mathematical operations on the given data and the output is provided by output neurons. Inputs follow a forward path where each input is multiplied by its corresponding synaptic weight and summed up. Sometimes a constant bias term is also added to weighted sum and then fed to the activation function to generate neuron output. For prediction problems nonlinear sigmoid transfer functions are used in hidden units and linear transfer function is used in output units. Here we label $P$ as input and $O$ as neuron output. Neuron output of one layer becomes the input to the next layer. From Equation (1), the ith parameter of input $P_{i}$ from unit $i$ is forwarded to unit $j$ of the network through network synaptic weight $W_{j i}$ and bias $b_{j}$ connected to jth unit.

$$
\text { net }_{j}=\sum_{i=1}^{N} W_{j i} P_{i}+b_{j}
$$

Because of logistic sigmoid transfer function in $j$ th unit, the summed weighted input is transformed into neuron output $O j$ by using suitable transfer function such as logistic sigmoid as given in Equation (2), and propagated forward as input $\left(O_{j}\right)$ to the neuron in the next layer.

$$
O_{j}=f\left(n e t_{j}\right)=\frac{1}{1+e^{-\left(n e t_{j}\right)}}
$$

After each forward iteration, predicted outputs $\left(O_{i j}\right)$ are compared with experimental (target) outputs $\left(T_{i j}\right)$ and an error is calculated as a mean square error (MSE):

$$
M S E=\frac{1}{N} \sum_{i=1}^{N}\left(T_{i j}-O_{i j}\right)^{2}
$$

where $N$ is the number of data points, $T_{i j}$ is the experimental (target) output and $O_{i j}$ is the network (predicted) output of data sets $i$ for the $j$ th neuron. 
If the generated error does not meet the user specified criteria the error will be propagated back to hidden units to update the weights and biases for the next iteration. This iterative process will continue until any user specified criteria is met. For the training of the neural network model, MATLAB ANN toolbox function 'trainbr' was used. It is an incorporation of the Levenberg-Marquardt (LM) algorithm and the Bayesian regularisation (Bayesian learning) into backpropagation to train the neural networks. Backpropagation algorithm calculates the Jacobian matrix of the performance function with respect to the weight and bias variables. Each variable is adjusted according to LM updated rule:

$$
\Delta W=\left(J^{T} J+\mu I\right)^{-1} J^{T} e
$$

Here $J$ is the Jacobian matrix of derivatives of each error to each weight, $\mu$ is the learning parameter and $e$ is the error vector, $I$ is identity unit matrix and superscript $T$ indicates matrix transposition. Bayes' rule automatically regulates the network's performance for smoother running to avoid any overfitting. Further discussion on LM algorithm and Bayesian regularisation is beyond the scope of this article, the interested reader may find detailed description in the literature [17-20].

Multilayer feed forward ANN with two hidden layers was trained using Matlab ANN toolbox function 'trainbr'. It consists of four neurons in the input layer (buffer neurons which perform no any mathematical task but just pass the input to first hidden layer neurons), three neurons in the first and second hidden layer each and one neuron in the output layer (i.e., 4-[3-3] $]_{2}-1$ ). Since there is no certain rule for selection of the number of layers and neurons in each layer, the above network structure was selected by trial and error method for all three models. Several networks with different layers, units in layer(s), training algorithms, training parameters and performance functions have been trained with the objective to minimise the training error and better generalisation on unseen data. The architecture and parameters of the selected network are given in Figure 1 and Table 2.

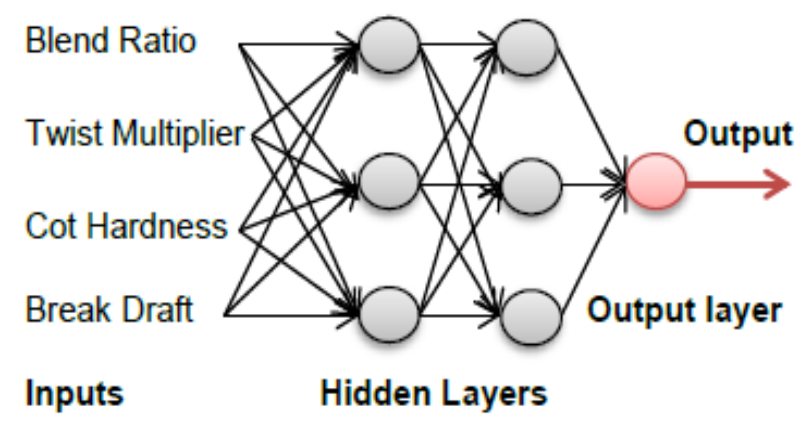

Figure 1. Architecture of ANN model

As backpropagation algorithm updates weights and biases by delta rule of derivative, differentiable transfer functions (activation function) are necessary in hidden layers. Tangent sigmoid and logistic sigmoid transfer functions were tested. They are bounded differentiable real functions defined for all real input values and have a positive derivative at each point; they squash infinite input range into finite output range.
Table 2. ANN parameters

\begin{tabular}{|c|c|}
\hline Parameters & Functions/values \\
\hline Network & $4-[3-3]_{2}-1$ \\
\hline Transfer functions & \{logsig, logsig, purelin $\}$ \\
\hline Inputs & 4 \\
\hline Output & 1 \\
\hline Learning rate & 0.08 \\
\hline$\mu$ & 0.7 \\
\hline Epochs & 1000 \\
\hline Performance goal & 0.01 \\
\hline Training function & trainbr \\
\hline
\end{tabular}

Finally on the basis of performance, logistic sigmoid was selected as the activation function for network training. Linear transfer function is used in the output unit of output layer, while mean square error (MSE) was used as a performance function. To analyse the network error in terms of actual units of the predicted property, an addition was made in the existing code of ANN to get error directly in terms of mean absolute error (MAE).

\section{Multiple Linear Regression}

The advantage of regression equations lies in the signs (+/-) of their coefficients which state the direction of effect of a particular variable. The MLR equations were developed using the experimental data. The developed equations were also validated with un-seen data and MAE was calculated for each model. Furthermore, the performance of multiple linear equations and ANNs were compared by considering the MAE and the coefficient of determination realised in both cases.

\section{Results and discussion}

\section{Predicting yarn evenness by ANN}

For neural network modelling, the experimental data was divided into two sets, training and test. Training set consists of 40 samples while remaining 8 data pairs were used for the model testing. For better generalisation of the model, experimental data was preprocessed into values between 0 and 1 , and after the completion of training it was then postprocessed into the original form by using appropriate Matlab codes. The performance of the trained model was judged on unseen data on the basis of a network coefficient of determination $\left(R^{2}\right)$ and MAE.

The prediction performance of the ANN on the training and unseen data is presented in Table 3 (yarn CVm\% column) and Figure 2. The line diagram of actual versus predicted values during network training is given in Figure 2 (a), it depicts that the predicted values approximate well to actual experimental values. The performance on unseen data is stated in Figure 2 (b) which shows that both lines are following almost the same 
path with a small deviation at the last two experiments which are in an acceptable range. It advocates that, there is a very strong correlation between the experimental and the neural network predicted (response) values.

The coefficient of determination $\left(R^{2}\right)$ states that 99 and $96 \%$ of the variability in predicted variable is explained by the explanatory input variables in the training and test sets, respectively. It is evident that very small variance has remained unexplained in the model by predictors. The reported mean absolute error, 0.14 and 0.26 on training and test sets, respectively; expressed in terms of $\mathrm{CVm} \%$ indicates the prediction power and precision of the ANN model. This shows the prominence of input variables used in the present model to predict yarn evenness, because while predicting blended yarn evenness including machine parameters, fibre blend ratio itself plays a vital role in determining yarn evenness. The relative importance of individual input variable will be discussed later in subsequent sections.

\section{Predicting yarn evenness by MLR}

MLR equation of yarn evenness (CVm\%) is given in Equation (5)

Yarn CVm\% $=7.98+2.17 X_{1}+0.21 X_{2}+0.06 X_{3}+0.17 X_{4}$

where $X_{1}$ is the PES/CO blend ratio, $X_{2}$ is the twist multiplier $\left(\alpha_{e}\right), X_{3}$ is the back roller cot hardness in degree shore, and $X_{4}$ is the break draft ratio.

Table 3. Performance of ANN models

\begin{tabular}{|c|c|c|c|c|c|c|}
\hline Parameters & \multicolumn{2}{|c|}{ Yarn CVm\% } & \multicolumn{2}{c|}{ Yarn Tenacity (cN/Tex) } & \multicolumn{2}{c|}{ Yarn Elongation (\%) } \\
\hline & Training set & Test set & Training set & Test set & Training set & Test set \\
\hline$R^{2}$ & 0.99 & 0.96 & 0.99 & 0.99 & 0.98 & 0.99 \\
\hline Error (\%) & 2.7 & 5.0 & 2.3 & 3.0 & 2.8 & 5.8 \\
\hline MAE & 0.14 & 0.26 & 0.20 & 0.27 & 0.12 & 0.26 \\
\hline
\end{tabular}
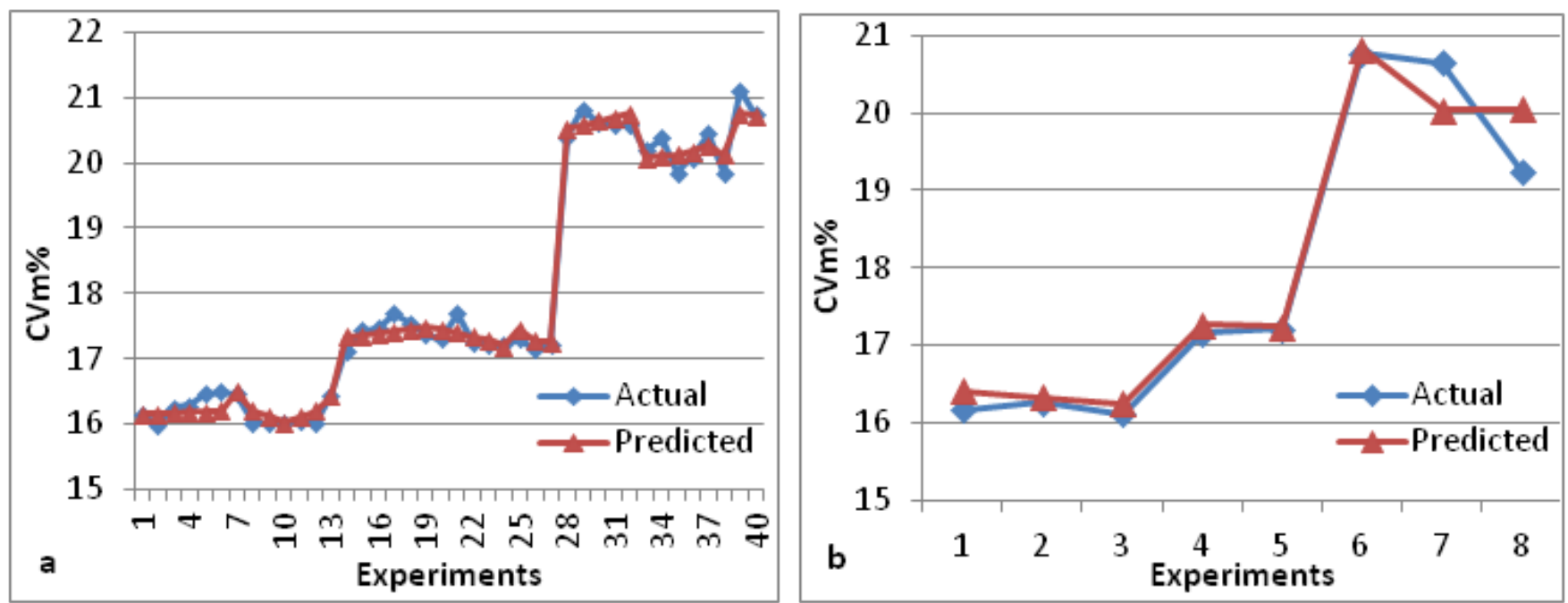

Figure 2. Performance of $A N N$ model for $\mathrm{CVm} \%$ prediction (a) training, (b) testing

Above equation confirms the positive correlation between independent and dependent variables. It states that with an increase in blend ratio (increase in cotton share), back roller hardness and break draft and twist multiplier, the yarn $\mathrm{CVm} \%$ increases. This is because cotton fibres are naturally nonuniform in length and diameter which causes nonuniformity in the yarn structure. Hence, CVm\% increases. Furthermore, back roller cot hardness also shows significant effect on blended yarn $\mathrm{CVm} \%$ due to reduced contact surface area of harder cots and hence reduced grip and guidance to fibres especially cotton fibres which need more control. This effect is more prominent in samples having a higher cotton fibre proportion [5]. Other input variables also have the same effect on yarn $\mathrm{CVm} \%$ but it is not significant as stated in Table 6 through standardised coefficients ( $ß$ coefficients).

The coefficient of determination $\left(R^{2}\right)$ and MAE of regression models are given in Table 4 (yarn $\mathrm{CVm} \%$ column). The values of $R^{2}$ and $R^{2}$ (adj) of model indicate that $91-92 \%$ of the variability in the predicted variable is explained by the explanatory input variables showing MAE of 0.4 . Whereas the performance of the developed model on new data is also better which explains $88 \%$ variance by model input variables with MAE of 0.6 in terms of $\mathrm{CVm} \%$ which is slightly higher than model error. By comparing Tables 3 and 4 (yarn CVm\% columns), it is obvious that the coefficient of determination $\left(R^{2}\right)$ of ANN model is greater than the regression model and the MAE of ANN is much lesser from developed regression model. It advocates that ANN is a better predictor of yarn evenness than MLR. 
Table 4. Performance of regression models

\begin{tabular}{|c|c|c|c|c|c|c|}
\hline Parameters & \multicolumn{2}{|c|}{ Yarn CVm\% } & \multicolumn{2}{c|}{ Yarn Tenacity (cN/Tex) } & \multicolumn{2}{c|}{ Yarn Elongation (\%) } \\
\hline & Model & Test & Model & Test & Model & Test \\
\hline $\mathrm{R}^{2}$ & 0.92 & 0.88 & 0.97 & 0.97 & 0.95 & 0.98 \\
\hline $\mathrm{R}^{2}(\mathrm{adj})$ & 0.91 & - & 0.97 & - & 0.95 & - \\
\hline $\mathrm{MAE}$ & 0.40 & 0.60 & 0.32 & 0.68 & 0.23 & 0.30 \\
\hline
\end{tabular}

\section{Predicting yarn tensile properties by ANN}

Yarn tenacity and elongation were modelled separately by following the same procedure as explained in the previous section. The prediction performance of ANN on network training and testing is given in Table 3 (yarn tenacity and yarn elongation columns), Figure 3 and Figure 4 . It has been observed that both models have generalised very well by showing that $99 \%$ variability in predicted variables in model as well as test set explained by the selected input variables and acceptable MAE of 0.27 and 0.26 , in terms of units of tenacity and elongation, respectively, during the test phase. It is also obvious from Figures 3 (a) and 4 (a) that both models have been trained very well, where line of predicted values shows the same trend like actual values. The same trend is depicted on seen data as stated in Figure 3 (b) and figure 4 (b).

These outcomes are the evidence of prediction power of neural networks to map complex interactions among yarn quality characteristics and machine and material variables and the selection of appropriate influencing input variables for the model.

The PES/CO material ratio has a significant influence on the blended yarn's tensile properties, because the characteristic properties of cotton and polyester fibres are different, therefore blended yarn's characteristics depends up on PES/CO ratios. Similarly, twist multiplier has a positive effect on the yarn tensile
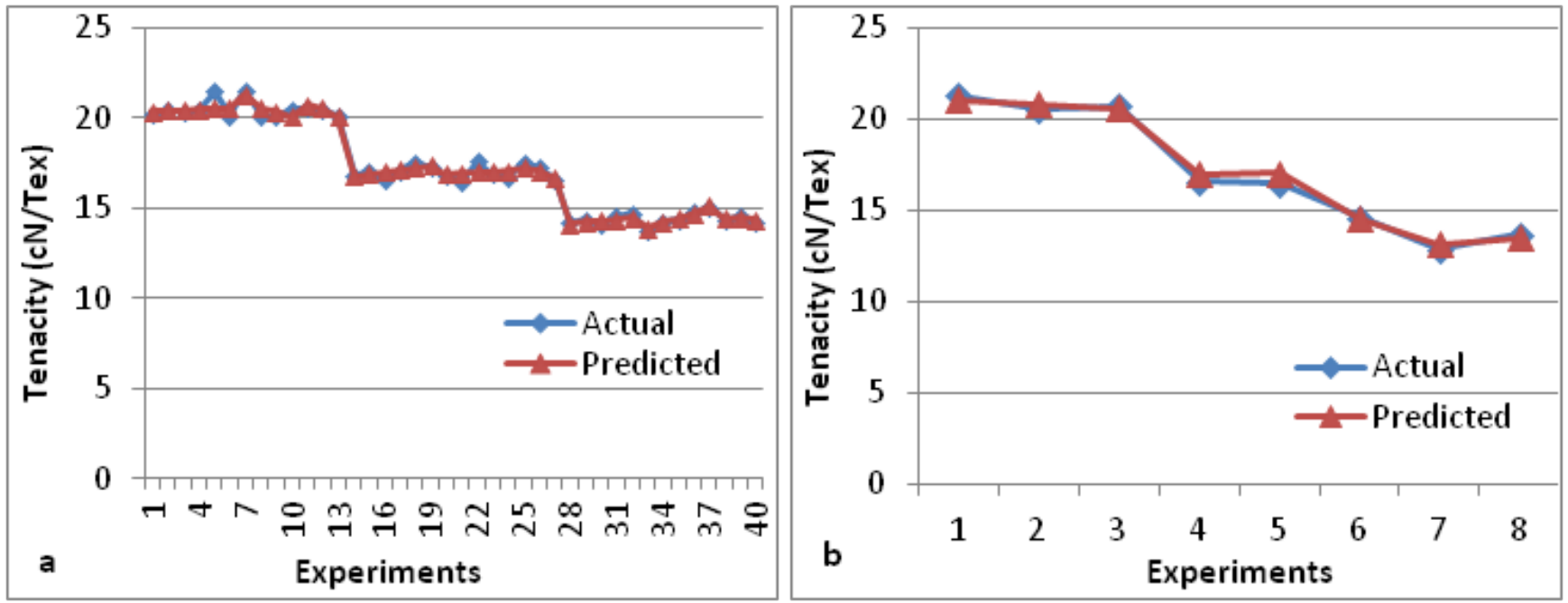

Figure 3. Performance of ANN model on yarn tenacity prediction (a) training, (b) testing
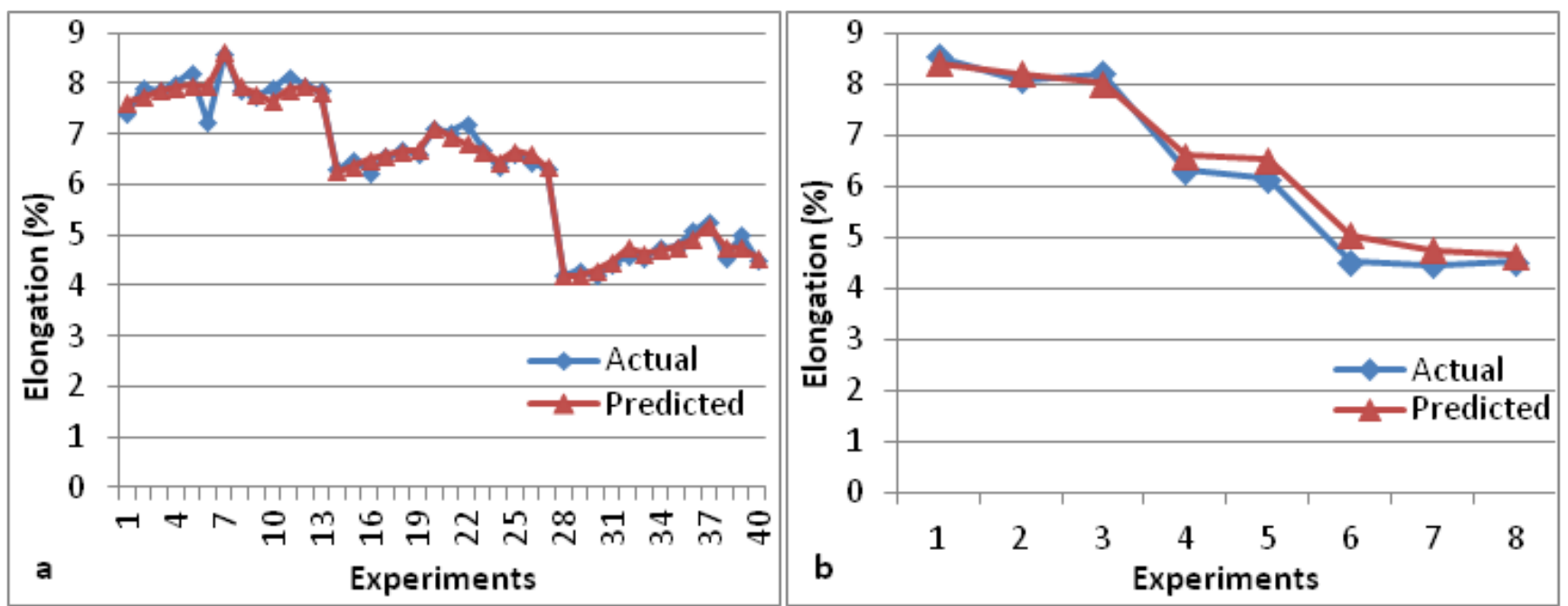

Figure 4. Test performance of ANN model for yarn elongation prediction (a) training, (b) testing 
properties up to spinning limit, beyond that its effect becomes negative, that is, tensile properties decrease. Furthermore, improper draft and roller hardness also have a negative influence on the yarn characteristics.

\section{Predicting yarn tensile properties by MLR}

The developed MLR equations of yarn tenacity and elongation are as follows:

$$
\begin{aligned}
& \text { Yarn Tenacity }\left(\frac{c N}{\text { Tex }}\right)=21.77-3.04 X_{1} \\
& +0.10 X_{2}-0.02 X_{3}+1.64 X_{4} \\
& \text { Yarn Elongation }(\%)=10.92-1.63 X_{1} \\
& -0.58 X_{2}-0.03 X_{3}+2.45 X_{4}
\end{aligned}
$$

where $X_{1}$ is the PES/CO blend ratio, $X_{2}$ is the twist multiplier $\left(\alpha_{e}\right), X_{3}$ is the back roller cot hardness in degree shore, and $X_{4}$ is the break draft ratio.

Equation (6) expresses that, by increasing cotton share and cot hardness yarn tenacity decreases, while tenacity increases with increase in twist multiplier and break draft. This is due to inherent characteristics of cotton fibres which are less strong as compared to polyester fibres and higher back roller cot hardness may lead to improper fibre control in predrafting zone which causes irregularities in yarn structure, consequently reduction in tenacity (also obvious from Eq. 5). The twist multipliers used in this study are within the spinning limits; therefore, they show a positive effect on the yarn tenacity. The break draft ratios used in this study comprised the whole available range of that particular machine. This reflects that for this particular yarn count, blend ratios, roving hank and twist multiplier, increase in break draft prepare the roving well for the main draft and consequently produces yarn with less thin places (which are the cause of yarn early failure), hence, tensile properties increase. Yarn elongation decreases with all variables except break draft ratio as shown in Equation (7) and discussed above.

Both modelling techniques, that is, ANN and MLR, are capable to predict yarn tensile properties, but ANN models predict tensile properties of yarn with a higher coefficient of determination $\left(R^{2}\right)$ and lesser MAE. This states that ANN models have a clear advantage over regression models developed in this study. On the basis of acquired outcomes discussed in this section, it is suggested that ANN is a very powerful predicting tool with higher prediction power as compared with MLR and can be applied for prediction of PES/CO blended yarn characteristics. Developed models are suitable for practical applications, especially for those new articles for which spinners have no previous experience. It saves time, material and labour costs. This research is only valid in the given knowledge domain.

\section{Analysis of relative importance of input variables}

To investigate the relative importance and contribution of input variables in the developed models, rank analysis is a widely practiced technique. In this technique predictors are ranked according to their influence on the stability of the model.

Relative importance of predictors is done by making one predictor at a time to zero in the optimised ANN model. The increase in MAE in test set was recorded for each predictor and the relative change in MAE was calculated in percentage with reference to the MAE of the optimised models. The inputs were ranked according to their relative importance; the higher the increase in percent mean absolute error the more important the variable. This technique is analogous to the input saliency test [21], in which one input at a time from the optimised model is eliminated. The results are expressed in Table 5. It is observed that removing one input or making it to zero at a time the same change in MAE is reported.

The rank analysis using ANN model in Table 5 reveals that in all three models, fibre blend ratio is the most important input variable. By adjusting it to zero in optimised ANN models, error increases enormously. This confirms the well-established fact, that increase in polyester share improves yarn uniformity and physical properties. It is because of uniform staple length and fineness as well as higher tenacity of polyester fibres. The second influential parameter according to rank analysis of optimised ANN model is break draft for yarn evenness and tenacity, whereas it is cot hardness for yarn elongation. It also confirms previous findings $[4,22]$ that proper break draft is important for roving preparation for main draft and to avoid drafting wave. Twist multiplier ranked third influential input parameter in optimised models of tenacity and elongation, whereas it is cot hardness in yarn evenness model. Except blend ratio, other predictors have varying rank

\begin{tabular}{|c|c|c|c|c|c|c|c|c|c|}
\hline \multirow{2}{*}{$\begin{array}{c}\text { Inputs } \\
\text { Excluded input } \\
\text { parameter }\end{array}$} & \multicolumn{3}{|c|}{ CVm\% } & \multicolumn{3}{|c|}{ Tenacity [cN/tex] } & \multicolumn{3}{|c|}{ Elongation [\%] } \\
\hline & $\begin{array}{l}\text { Mean } \\
\text { absolute } \\
\text { error }\end{array}$ & $\begin{array}{c}\% \\
\text { Increase } \\
\text { in MAE }\end{array}$ & Rank & $\begin{array}{l}\text { Mean } \\
\text { absolute } \\
\text { error }\end{array}$ & $\begin{array}{c}\% \\
\text { Increase } \\
\text { in MAE }\end{array}$ & Rank & $\begin{array}{l}\text { Mean } \\
\text { absolute } \\
\text { error }\end{array}$ & $\begin{array}{c}\% \\
\text { Increase } \\
\text { in MAE }\end{array}$ & Rank \\
\hline Blend ratio & 1.70 & 553.8 & 1 & 2.74 & 914.8 & 1 & 1.39 & 434.6 & 1 \\
\hline Twist multiplier & 0.32 & 23.1 & 4 & 0.65 & 140.7 & 3 & 0.33 & 26.9 & 3 \\
\hline Cot hardness & 0.35 & 34.6 & 3 & 0.56 & 107.4 & 4 & 0.34 & 30.8 & 2 \\
\hline Break draft & 0.38 & 46.2 & 2 & 0.66 & 144.4 & 2 & 0.29 & 11.5 & 4 \\
\hline
\end{tabular}
in the three different models, but the difference between them is not significant.

Table 5. Relative importance of input variable using ANN model 
The relative importance of predictor variables of developed regression models was analysed by using standardised coefficients or $B$ coefficients of input variables. Although a lot of criticism is available in statistical scientific literature on the use and correctness of this method, $B$ coefficients are widely used for variable selection, comparing the effect by changing variables and relative importance measurement. This may be due to the unavailability of alternative solutions [23]. Standardisation is done by subtracting variable's mean from each of its value and then dividing the new value with standard deviation of the variable. As the variance of each variable is 1 , $B$ coefficient reflects how much standard deviation of response will change per standard deviation increase in the predictor variable. As the standardised coefficients have no unit, it makes comparison easy. The higher the value of $B$ coefficient the more important is the variable, that is, the variable has more influence on the dependent variable. The relative contribution $\left(R_{c} \%\right)$ of the ith variable from $N$ number of variables can be calculated as follows:

$$
R_{c} \%=R^{2}\left(\frac{\beta_{i}}{\sum_{i=1}^{N} \beta_{i}}\right) \times 100
$$

In this study the relative importance of the input variables is determined in the form of rank, as was done for ANN models to make comparison of both modelling techniques more realistic.

MLR rank analysis is shown in Table 6. Similar to ANN rank analysis, blend ratio is clearly ranked number 1 for all three MLR models, it is also evident from the P-values of developed MLR models and their variables (Table 7) - which reflect the statistical significance of the models and predictor variables. Whereas back roller cot hardness, twist multiplier and break draft ratio are ranked accordingly as stated in Table 6 , but within group difference is not significant.
The probable reasons of influence of different input variables are already explained. While comparing ranks of ANN and MLR models, except blend ratio which is highly significant variable, other variables have almost different ranks in ANN and MLR models, but as the difference between $B$ coefficients within MLR models and change in error in optimised ANN model is not significant, therefore it can be inferred that, the ranks of predictors are almost the same for both models.

\section{Conclusions}

In the present study, blended yarn evenness in terms of $\mathrm{CVm} \%$ and tensile properties was successfully predicted with ANN and MLR models by using PES/CO blend ratio, twist multiplier, back roller covering hardness and break draft ratio as input variables. The results reveal that both modelling tools have excellent prediction capabilities for PES/CO yarn evenness, tenacity and elongation.

The coefficient of determination $\left(R^{2}\right)$ of all ANN models expresses 98-99\% variability in the predicted variables explained by the explanatory variables is greater than corresponding regression models. While the MAE in terms of output variables of ANN models is significantly lower than the corresponding regression models. Therefore, it can be asserted that artificial neural network is a more powerful prediction tool as compared with MLR technique.

Rank analysis was also performed to determine the relative importance of input variables of optimised ANN models and standardised MLR models. It states that in all models, fibre blend ratio is the most important input variable, whereas, other predictors have varying rank in different models. While comparing ranks of ANN and MLR models, except blend ratio

Table 6. Relative importance of input variable for MLR model

\begin{tabular}{|c|c|c|c|c|c|c|}
\hline \multirow{2}{*}{ Input parameters } & \multicolumn{2}{|c|}{ Yarn CVm\% } & \multicolumn{2}{c|}{ Yarn Tenacity (cN/Tex) } & \multicolumn{2}{c|}{ Yarn Elongation \% } \\
\cline { 2 - 6 } & B Coefficient & Rank & B Coefficient & Rank & B Coefficient & Rank \\
\hline Blend ratio [-] & 0.982 & 1 & -0.983 & 1 & -0.963 & 1 \\
\hline Twist multiplier [-] & 0.028 & 3 & 0.010 & 4 & -0.101 & 2 \\
\hline Back roller hardness [ ${ }^{\circ}$ Sh] & 0.103 & 2 & -0.020 & 3 & -0.075 & 4 \\
\hline Break draft [-] & 0.005 & 4 & 0.035 & 2 & 0.096 & 3 \\
\hline
\end{tabular}

Table 7. P-values of regression models

\begin{tabular}{|c|c|c|c|}
\hline \multirow{2}{*}{ Inputs } & \multicolumn{2}{|c|}{ P-values } \\
\cline { 2 - 4 } & CVm\% & Tenacity [cN/tex] & Elongation [\%] \\
\hline Model & 0.03 & 0.00 & 0.00 \\
\hline Blend ratio & 0.00 & 0.00 & 0.00 \\
\hline Twist multiplier & 0.55 & 0.74 & 0.01 \\
\hline Cot hardness & 0.04 & 0.52 & 0.05 \\
\hline Break draft & 0.92 & 0.24 & 0.01 \\
\hline
\end{tabular}


other variables have almost different ranks in ANN and MLR models. Therefore, it can be inferred that in ANN and MLR models, the input variables not necessarily have the same contribution for prediction of the same property, but this should be noted that within differences in contributions of other three variables are not significant. The performance of developed models also supports our conversion and the selection criteria of dummy variable factors.

\section{References}

[1] Grosberg, P., lype, C. (1999). Yarn Production: Theoretical Aspects (1st ed.). The Textile Institute Manchester England.

[2] Nawaz, S. M., Shahbaz, B., \& Yousaf, C. K. (1999). Effect of different blends with various twist factors on the quality of P/C blended yarn. Pakistan Textile Journal, 48(6), 2629.

[3] Basu, A. (2009). Yarn structure-properties relationship. Indian Journal of Fiber and Textile Research, 34(3), 287299.

[4] Malik, S. A.; Tanwari, A., Syed, U., Qureshi, R. F.; \& Mengal, N. (2012). Blended Yarn Analysis: Part I-Influence of Blend Ratio and Break Draft on Mass Variation, Hairiness, and Physical Properties of 15 Tex PES/CO Blended RingSpun Yarn. Journal of Natural Fibers, 9(3), 197-206.

[5] Malik, S. A.; Mengal, N., Saleemi, S., \& Abbasi, S. A. (2013). Blended Yarn Analysis: Part II - Influence of Twist Multiplier and Back Roller Cot Hardness on Mass Variation, Hairiness, and Physical Properties of 15 Tex PES/COBlended Ring-Spun Yarn. Journal of Natural Fibers, 10(3), 271-281.

[6] Veit, D.; Hormes, I., Bergmann, J., \& Wulfhorst, B. (1996). Image processing as a tool to improve machine performance and process control. International Journal of Clothing Science and Technology, 8(1/2), 66-72.

[7] Farooq, A.; \& Cherif, C. (2008). Use of Artificial Neural Networks for Determining the Leveling Action Point at the Auto-leveling Draw Frame. Textile Research Journal, 78(6), 502-509.

[8] Farooq, A.; \& Cherif, C. (2012). Development of Prediction System Using Artificial Neural Networks for the Optimization of Spinning Process. Fibers and Polymers, 13(2), 253-257.

[9] Cheng, L.; \& Adams, D. L. (1995). Yarn strength prediction using neural networks part l: fiber properties and yarn strength relationship. Textile Research Journal, 65(9), 495- 500 .
[10] Üreyen, M. E.; \& Gürkan, P. (2008). Comparison of Artificial Neural Network and Linear Regression Models for Prediction of Ring Spun Yarn Properties. I. Prediction of Yarn Tensile Properties, Fibers and Polymers, 9(1), 87-91.

[11] Üreyen, M. E.; \& Gürkan, P. (2008). Comparison of Artificial Neural Network and Linear Regression Models for Prediction of Ring Spun Yarn Properties. II. Prediction of Yarn Hairiness and Unevenness. Fibers and Polymers, 9(1), 92-96.

[12] Demiryürek, O.; \& Koç, E. (2009). Predicting the Unevenness of Polyester/Viscose Blended Open-end Rotor Spun Yarns Using Artificial Neural Network and Statistical Models. Fibers and Polymers, 10(2), 237-245.

[13] Demiryürek, O.; \& Koç, E. (2009). The Mechanism and/ or Prediction of the Breaking Elongation of Polyester/ Viscose Blended Open-end Rotor Spun Yarns. Fibers and Polymers, 10(5), 694-702.

[14] Ramesh, M.C.; Rajamanickam, R; Jayaraman,S. (1995). The Prediction of Yarn Tensile Properties by Using Artificial Neural Network. Journal of Textile Institute, 86(3), 459469.

[15] Beltran, R.; Wang, L.; \& Wang, X. (2004). Predicting Worsted Spinning Performance with an Artificial Neural Network Model. Textile Research Journal, 74(9), 757-763.

[16] Pynckels, F.; Kiekens, P.; Sette, S.; Van Langenhov, L.; Impe, K. (1995). Use of Neural Nets for Determining the Spinnability of Fibres. Journal of Textile Institute, 86(3), 425-437.

[17] Wilamowski, B. M.; Iplikci, S.; Kaynak,O.; and Efe, M. Ö., (2001). An algorithm for fast convergence in training neural networks. Proceedings of International Joint Conference on Neural Networks, Washington, DC USA, 3, 1778-1782

[18] Fine, T. L.(1999). Feedforward Neural Network Methodology, Springer Verlag, New York USA.

[19] MacKay, D. J. C. (1992). Bayesian Interpolation. Neural Computation, 4(3), 415-447.

[20] Dan Foresee, F.; Hagan, M. T. (1997). Gauss-newton approximation to Bayesian learning. Proceedinds of the International Joint Conference on Neural Networks, 3, 1930-1935.

[21] Majumdar, P. K.; Majumdar, A. (2004). Predicting the Breaking Elongation of Ring Spun Cotton Yarns Using Mathematical, Statistical, and Artificial Neural Network Models. Textile Research Journal, 74(7), 652-655.

[22] Klein, W. (1987). A Practical Guide to Ring Spinning: Short-staple spinning series: manual of textile technology (1st ed.) The Textile Institute Manchester England.

[23] Bring, J. (1994). How to Standardize Regression Coefficients. The American Statistician, 48(3), 209-213. 